\title{
Traduire
}

Une eutre perspective sur $r$ tatadciction

Revue française de la traduction

$242 \mid 2020$

Passons au vert

\section{La concision en traduction scientifique : une valeur ajoutée}

Richard Ryan

\section{(2) OpenEdition}

1 Journals

Édition électronique

URL : http://journals.openedition.org/traduire/2052

DOI : 10.4000/traduire.2052

ISSN : 2272-9992

Éditeur

Société française des traducteurs

Édition imprimée

Date de publication : 30 juin 2020

Pagination : 113-125

ISSN : 0395-773X

\section{Référence électronique}

Richard Ryan, «La concision en traduction scientifique : une valeur ajoutée », Traduire [En ligne], 242 |

2020, mis en ligne le 15 juillet 2020, consulté le 21 décembre 2020. URL : http://

journals.openedition.org/traduire/2052 ; DOI : https://doi.org/10.4000/traduire.2052 


\section{La concision en traduction scientifique : une valeur ajoutée}

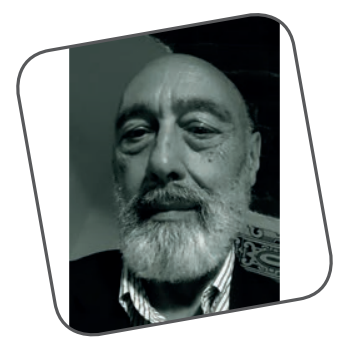

\section{Richard Ryan}

"Weniger ist mehr.»

Ludwig Mies van der Rohe

«Précision, clarté et concision»: rédacteurs et traducteurs scientifiques (les termes désignant les personnes sont au masculin dans un souci de... concision) connaissent bien cette triple injonction que les manuels de bonnes pratiques et autres style guides leur rappellent sans cesse. Précision, car dans les sciences dites exactes, un texte approximatif ou peu intelligible risque d'être inexploitable ou trompeur - rappelons en passant que cette précision concerne non seulement la terminologie, mais aussi la modalité (nécessité, probabilité, capacité, obligation) et la relation (dépendance, significativité, corrélativité, sélectivité, etc.). Clarté, car la bonne articulation du raisonnement déductif ou inductif qui sous-tend le discours scientifique détermine sa valeur performative. Concision, car seuls comptent les faits observés - les phénomènes - et le raisonnement qui les ordonne et qui 
les interprète. Un texte scientifique transmet et commente des connaissances validées par des constats; son auteur ne cherche pas à séduire, si ce n'est par la justesse de sa démonstration, par la nouveauté ou la portée de ses conclusions, dont il est pressé d'informer son auditoire par la voie la plus directe.

Précision, clarté et concision ne sont pas pour autant des variables indépendantes dont la simple somme arithmétique serait une mesure de la qualité formelle d'un texte scientifique. La précision est vitale, la clarté est importante, la concision est utile: elle facilite la transmission en permettant un accès plus rapide, plus direct et plus sûr aux informations comme aux arguments. On dit que la concision rend le document plus efficace, en s'en tenant à l'aspect pratique: sa fonction référentielle consistant, de fait, à assurer la bonne diffusion des contenus dénotés. La concision aide l'utilisateur à appréhender rapidement des faits ou des arguments nouveaux, à suivre un cheminement intellectuel sans trébucher, à décider de manière éclairée, à prendre connaissance d'un protocole ou d'un mode opératoire.

Paradoxalement, par son effet de condensation, la concision peut parfois sembler aller à l'encontre de la clarté. Trop de concision peut obscurcir, la compacité invite à l'opacité. Mais la concision peut aussi produire un effet immédiat et puissant, comme un alcool avalé sec - un distillat, un concentré dont la densité seule peut rendre un énoncé incisif ou tranchant, et donc plus persuasif (à défaut d'être plus convaincant). La concision est en soi péremptoire, qualité peu recommandée dans un contexte purement scientifique, de sorte que, loin d'être l'auxiliaire de la précision ou de la clarté, elle peut distraire, au plein sens du terme - divertir et détourner. Mais déclinons d'abord les différentes fonctions de la concision. Nous en distinguerons trois ici, que nous appellerons référentielle, conative et phatique, en référence au schéma classique de Roman Jakobson (1963). Nous verrons ensuite en quoi ces considérations intéressent le traducteur scientifique.

\section{La concision référentielle}

À l'écrit, la concision a toujours joui d'un préjugé favorable: en France, Bossuet, La Bruyère et Montesquieu sont salués 
comme des maîtres de la concision, comme l'ont été, dans un autre registre, les rédacteurs du Code Napoléon. En langue anglaise, pour les célèbres prescripteurs Henry et Francis Fowler, auteurs de The King's English (1908), un ouvrage précurseur de tous les manuels de style britanniques, le bon style est «direct, simple, brief, vigorous and lucid». L'Américain William Strunk précise quant à lui dans le classique Elements of Style, dont la première édition date de 1920:

Vigorous writing is concise. A sentence should contain no unnecessary words, a paragraph no unnecessary sentences, for the same reason that a drawing should have no unnecessary lines and a machine no unnecessary parts. This requires not that the writer make all his sentences short, or that he avoid all detail and treat his subjects only in outline, but that every word tell.

Cette définition privilégie la notion d'économie de moyens, de parcimonie, en même temps qu'elle introduit celles d'efficacité, de concentration: tous les mots doivent porter. Moins de mots donc, mais des mots qui parlent.

Dans les sciences exactes, la frugalité - suppression des redondances, abréviation, resserrement, contraction - soutient la clarté lorsqu'elle rend une démonstration «élégante», dans l'acception donnée par le Littré: "Se dit de calculs ou de constructions qui sont à la fois simples et ingénieuses.» La solution d'un problème scientifique se veut simple et ce qui est simple doit s'exprimer sobrement, comme une grande vérité.

Si le souci d' «aller à l'essentiel» est toujours louable, trop de concision peut desservir la clarté lorsqu'elle rend trop implicite ce qui était explicite (par l'ellipse), ou qu'elle généralise à l'excès. Il en résulte une charge cognitive malvenue pour le lecteur non initié (ou fatigué). C'est la concision du raccourci hâtif. Elle peut s'opposer à l'exhaustivité et refuser la périphrase éclairante. Un raisonnement complexe demande parfois un long développement à petits pas, exemples à l'appui. Même la répétition peut se justifier lorsqu'elle sert à faire ressortir ou à rappeler un élément important ou encore à lever une équivoque. Il importe donc de ne pas confondre concis et sommaire, condensé et tronqué, dense et impénétrable.

L'expression extrême de la concision est le sigle, très utilisé par les scientifiques, et source de difficultés pour le 
traducteur. Le sigle, défini à sa première occurrence, ou dans une liste d'abréviations, permet de réduire la longueur d'un texte par un effet mécanique et d'en accélérer la lecture.

Le souci de «faire porter les mots»évoqué par Strunk (1920) nous amène à considérer la concision dans sa fonction conative, appelée aussi impressive ou appellative, où la seule brièveté d'un énoncé a une influence sur le destinataire, effet qui peut être heureux ou au contraire inopportun, voire préjudiciable selon le contexte.

\section{La concision conative}

Au commencement était l'apostrophe. Puis vint le cri de guerre. Celui-ci trouve une nouvelle expression dans nos slogans politiques: de Montjoie! ou Hourra Staline! nous sommes passés à L'écologie debout! et En marche pour l'Europe! Mais le principe demeure: poussée à l'extrême, la concision dépouille l'énoncé de son contenu dénotatif, jusqu'à le rendre énigmatique, pour renforcer la puissance évocatrice et surtout mobilisatrice de la formule. C'est le prêt-à-scander.

Dans nos chroniques, l'exemple princeps de la concision conative, ou "formule magique», serait le fiat lux de la Création, devenu plus tard la devise de plusieurs universités nord-américaines (et le nom que le détective de fiction Nestor Burma a donné à son agence parisienne). Les Spartiates, réputés hommes de peu de mots, affectionnaient les formules brèves et incisives, que l'on a appelées plus tard laconismes. "Viens les prendre!»lance Léonidas à Xerxès qui lui demande de déposer les armes contre la vie sauve lors de la bataille des Thermopyles (c'est sans doute dans le même état d'esprit que le général Cambronne prononce son fameux mot à Waterloo). Ces paroles, parce que brèves, sont mémorables dans tous les sens du terme. Les apophtegmes des grands hommes recueillis par Plutarque ou les maximes des Sept sages de Grèce ont été conservés en raison de leur concision. La puissance conative de la forme courte s'exerce aujourd'hui dans le discours publicitaire: "Buvez. Éliminez» (Vittel), et dans les exhortations des dirigeants: "Get Brexit done!» (Boris Johnson). Enfin, dictons, proverbes, devises, adages, aphorismes et autres sentences nous proposent des sortes de concentrés de sagesse auxquels la 
concision parfois sibylline confère une autorité sans appel. Observons la fécondité de ce procédé même aujourd'hui, notamment en anglais: no pain, no gain; it takes one to know one; you do the crime, you do the time; use it or lose it; if you can't beat 'em, join 'em; there's no such thing as a free lunch; lads need dads sont des réalisations récentes.

Un stade avancé de la concision conative est l'acronyme, depuis l'ichthus des premiers chrétiens, au KISS! (keep it short and simple!). Mais son stade ultime est le silence, accusateur, pudique, bravache, buté, furieux ou indifférent.

La fonction conative de la concision se distingue de sa fonction phatique, même si les deux peuvent se recouper. Au sens strict, la fonction phatique concerne uniquement la relation entre l'émetteur et le destinataire. II ne s'agit cette fois ni de transmettre des connaissances ni de mettre en action, mais de susciter, de créer ou de renforcer les relations sociales.

\section{La concision phatique}

Il est bon de savoir parler pour ne rien dire, à condition d'être bref. Appelée en anglais "small talk», la conversation banale ne doit ennuyer ni l'émetteur ni le destinataire. Plus le contenu est maigre, plus le style doit être vif et enlevé. Dans les siècles passés, la concision trouvait son expression à travers le trait d'esprit ou «saillie», dont l'originalité et la virtuosité étaient les qualités premières. Ces bons mots, phrases assassines et propos mordants ou «lestes» étaient appréciés à la cour ou dans les salons, où leurs auteurs rivalisaient devant un public de connaisseurs. Forme avant tout orale et éphémère, le mot d'esprit a pourtant inspiré quantité de recueils et de nombreux sites web y sont aujourd'hui consacrés. La concision phatique s'emploie encore dans les titres des journaux, notamment ceux des tabloids britanniques, titres qui renseignent peu sur le contenu des articles qu'ils annoncent, mais qui accrochent le lecteur par un effet de connivence autour d'un jeu de langage partagé.

La fonction phatique de la concision se trouve dans l'accroche, ou "catchphrase» facile à retenir et qui active une association d'idées chez les initiés: " $\dot{A}$ demain, si vous le voulez bien» (Lucien Jeunesse, France Inter, 1965-1995). De même, les incipit ou les refrains des chansons populaires: "Sur l'écran 
noir de mes nuits blanches...»; "Ce soir, j'attends Madeleine...»; «Paroles, paroles, paroles...»; "I did it my way...», etc.

Tout comme la concision conative, la concision phatique trouve son ultime expression dans le silence, mais le silence complice.

La concision phatique cherche à créer une proximité entre l'auteur et le destinataire par des références ou des postures partagées. Si cette proximité, même factice, peut aider la communication, c'est en faisant entrer la subjectivité dans l'échange, ce qui est réputé incompatible avec le discours scientifique (quoique possible dans la vulgarisation scientifique, un tout autre genre).

\section{La concision en français et en anglais}

Les différentes langues n'attachent pas la même importance à la concision. En français, la clarté a longtemps été une vertu cardinale, non seulement du rédacteur mais, selon certains, de la langue elle-même: "Ce qui n'est pas clair n'est pas français; ce qui n'est pas clair est encore anglais, italien, grec ou latin» (Rivarol, 1784); "Un des principaux caractères de cette langue c'est d'abord son extrême clarté qui la rend moins susceptible qu'aucune autre, d'obscurité, d'équivoques et de double sens» (Allou, 1828). De nombreux ouvrages en ont fait l'éloge, tel que La Clarté française d'Antonin Vannier (1923). Quoi qu'il en soit, la tradition du discours délibératif, très présente dans la pratique scolaire, enjoint le rédacteur français à prendre le plus grand soin à ordonner le raisonnement du texte (c'est la dispositio de la rhétorique antique). Les articulations - énumérations, syllogismes, conditions, concessions, précisions, etc. - sont explicitées par des opérateurs logiques: d'abord... ensuite... enfin; si... alors; or... donc; d'une part... d'autre part; même si..., en effet..., en revanche...; certes...; toutefois...; par ailleurs...; en somme...; finalement... Ces multiples liaisons sont caractéristiques du texte scientifique français, à la différence du texte anglais qui avance par juxtaposition et parataxe, et où l'ordre textuel est l'ordre à la fois temporel et logique.

L'auteur français accompagne son lecteur. En anglais, le lecteur croit connaître déjà le chemin. Comme le touriste impatient qui boude les explications du guide, ou bien le client 
averti qui fuit le vendeur zélé, il préfère qu'on le laisse faire. Si l'on y ajoute la conceptualisation qui préfère le groupe nominal au verbe (par exemple "effectuer une analyse» plutôt qu' «analyser», «assurer la direction de»et non «diriger»), et ce que l'on pourrait appeler «l'étoffement euphonique» qui lisse les aspérités et régule le débit, il devient difficile en français de présenter un raisonnement développé qui soit concis sans être haché ou heurté. Dans les textes scientifiques, l'explicitation est ainsi la valeur par défaut qui en français l'emporte généralement sur la concision. Au contraire, la concision est une condition nécessaire (quoique non suffisante) à la qualité des écrits scientifiques rédigés en anglais, où l'on ne doit pas faire piétiner le lecteur par un excès de prévenance.

L'exhortation à la concision est omniprésente dans les style guides et instructions for authors: "A sentence should contain no unnecessary words, a paragraph no unnecessary sentences», écrit Strunk (1920). Pour George Orwell (1946), "If it is possible to cut a word out, always cut it out». "One of the most common flaws in research writing is its wordiness", affirme la Canadian Health Services Research Foundation dans ses consignes destinées aux rédacteurs. "Cut and cut again.»

Les guides de rédaction des travaux universitaires (academic writing) sont usuels dans les universités anglo-saxonnes. La concision y constitue toujours une rubrique importante. Voici une liste représentative de conseils en la matière:

- Eliminate redundant/ unnecessary/ padding/ filler words,

- Rephrase wordy constructions,

- Avoid repetition,

- Replace a phrase with a word,

- Get rid of adverbs and reduce your adjectives,

- Use the shortest synonym.

- Don't refer back.

Il existe de nombreuses formules unissant fond et forme pour rappeler à l'ordre le rédacteur indiscipliné: "Brevity is the soul of wit» (Hamlet, William Shakespeare); "Never use two words when one will do" (Thomas Jefferson); if in doubt, leave it out; stick to the facts; get to the point, etc.

Nos outils informatiques d'aide à la rédaction (proofing tools) intègrent cette injonction: en anglais, le correcteur grammatical de MS Word réprouve les locutions telles que 
with the exception of (except), take into account (consider), as a way to (to), a majority of (most), ou encore in particular, actually, quite (à supprimer), avec la recommandation "consider using concise language».

La recherche de la concision apparaît parfois comme une obsession dans l'anglosphère, comme a pu l'être la célébration de la clarté dans le monde francophone. Ces recommandations sont largement suivies par les rédacteurs professionnels anglophones au point d'être devenues un premier réflexe. Le rédacteur français, en revanche, cherchera la clarté d'abord dans l'articulation (gare à la phrase «boiteuse») et dans la cohérence.

\section{Et le traducteur scientifique, alors?}

La concision du texte scientifique est un critère de conformité stylistique, surtout en anglais. Mais la portée de ce critère se trouve prolongée par une nécessité éditoriale: celle de faire tenir une grande quantité de science dans un volume limité. Dans ce qui suit, nous ferons référence à l'écrit scientifique type qu'est l'article de recherche primaire (primary research paper) ou article IMRaD (pour Introduction, Materials and Methods, Results and Discussion), source de revenus importante pour certains traducteurs scientifiques, car devant presque toujours être publié en anglais. Dans les sciences exactes, de nombreux chercheurs non anglophones sont aujourd'hui à même de rédiger un article de recherche dans un anglais compréhensible, mais il est courant de faire appel à un traducteur-réviseur afin que le texte soit accepté plus facilement par les comités de lecture des grandes revues. La longueur d'un article de recherche est limitée pour des raisons de rentabilité éditoriale. Pour agréer au comité de lecture, l'article doit apporter des connaissances nouvelles, pertinentes et validées, du moins provisoirement, par des indices probants. Cette contrainte oblige les auteurs à un exercice de concision qui dépasse le simple style épuré recommandé pour tout texte scientifique. L'article de recherche est comme une valise dans laquelle on essaie de faire tenir trop d'affaires. On trie, on écarte le superflu, on la remplit avec méthode. Elle doit fermer sans forcer et ne rien contenir d'importance secondaire, tout en gardant un peu de 
place pour les ajouts de dernière minute: une nouvelle donnée expérimentale, un graphique explicatif ou une référence bibliographique.

Il arrive fréquemment que le rédacteur d'un article demande expressément à un traducteur-réviseur de "réduire la sauce», pour que son texte reste dans les limites de longueur prescrites par l'éditeur. Même quand la longueur du texte est conforme, les reviewers (membres du comité de lecture) trouvent souvent "difficult to read» un article rédigé dans un anglais trop étroitement calqué sur le français, ou sur-traduit, et demandent à l'auteur de le retravailler dans le sens d'une formulation plus concise. Ce travail de condensation est une prestation à part entière, liée à la traduction, mais relevant plutôt de la "paratraduction». Même en l'absence d'une demande formalisée, il est de bonne pratique de «décrémenter» un article écrit en français, sur-traduit en anglais, ou rédigé directement dans un anglais trop marqué par les habitudes rédactionnelles du français. Mieux vaut prévenir que guérir. On estime qu'un texte français est plus long de $10 \%$ en moyenne que son équivalent anglais. C'est le coefficient de foisonnement, lié aux propriétés constitutives des langues. En traduction scientifique, ce coefficient, plus élevé en raison des normes et pratiques de l'édition, peut facilement atteindre les $20 \%$.

La condensation peut s'opérer à différents niveaux, depuis une tournure jusqu'au texte dans sa totalité (par exemple en supprimant des opérateurs logiques ou des redites), en passant par le membre de phrase et la phrase, sans qu'il ne soit ni possible ni utile d'en établir une typologie. C'est une démarche opportuniste qui s'apparente souvent plus au grignotage, en gagnant un mot ici et là, qu'à la reformulation.

Voici quelques exemples attestés en guise d'illustration (auteurs et traducteurs resteront anonymes, déontologie oblige). Le lecteur angliciste reconnaîtra les procédés linguistiques répertoriés par les incontournables Vinay et Darbelnet dans leur Stylistique comparée du français et de l'anglais (1958). Nous n'en dirons rien, mais chercherons à relever plutôt l'effort de "dégraissage» (ou streamlining) qui produit des énoncés bien plus brefs que l'original. 


\section{Exemple 1}

«Les emplois se concentraient majoritairement dans des aires urbaines qui n'en finissaient pas de s'étendre avec le développement conjoint des zones d'activités et de l'habitat individuel pavillonnaire, très consommateurs en sol foncier.»

Traduction concise: "Most jobs were in urban areas, which spread further with the combined growth of land-intensive business parks and low-density housing."

Notons l'emploi des adjectifs land-intensive, bâtis selon une structure permettant souvent, par sa densité, un gain de concision par rapport au français (voir N-friendly. $N$-prone, $N$-ready, N-compliant, N-resistant/ proof, N-enriched, $\mathrm{N}$-enabled, N-based, N-driven, etc., où $\mathrm{N}$ est un nom commun), ainsi que low-density (voir sur le même modèle fixedrate, cut-price, variable-speed, fast-action, slow-release, etc.).

\section{Exemple 2}

"Pour les énergies intermittentes, comme l'éolien ou le solaire photovoltaïque, dont la production n'est souvent pas en phase avec la demande, le stockage est indispensable, sinon il faut utiliser des sources d'énergie complémentaires polluantes pour produire de l'électricité lorsqu'elles n'en génèrent pas.»

Traduction concise: "For intermittent energy production (wind-generated or solar photovoltaic), supply is often out of step with demand. Without storage, stand-in polluting energy sources must make up for shortfalls.»

Notons que la phrase française comporte une proposition relative incise (dont...) et une hypothèse négative (sinon...), alors que la traduction en anglais comporte deux phrases affirmatives. Le lien logique entre les deux phrases est implicite. Le traducteur estime que le lecteur rétablira le lien sans effort, que le rapport est «évident».

\section{Exemple 3}

"Des études ont montré que le céfuroxime présente une stabilité inférieure à $24 \mathrm{~h}$ à $25^{\circ} \mathrm{C}$. La réfrigération augmente la durée de stabilité mais les durées de conservation 
annoncées à 28 jours montrent tout de même l'apparition de produits de dégradation.»

Traduction concise: "We know that cefuroxime is stable for less than $24 \mathrm{~h}$ at $25{ }^{\circ} \mathrm{C}$. Refrigeration increases storage life but reported maximum storage times of 28 days still cause degradation products to form.»

Le texte français comporte douze substantifs et quatre verbes, contre cina substantifs dont trois composés nomnom (gain de densité) et cinq verbes en anglais. Le traducteur a préféré still à all the same pour «tout de même». Le texte en anglais est plus dense, mais encore lisible.

\section{Exemple 4}

"Le corollaire de cette évolution est l'accroissement des déplacements pendulaires.»

Traduction concise: "This trend means more commuting."

Traduction très concise: "Hence more commuting."

Il appartient au traducteur d'apprécier le degré de concision qui permettra d'apporter la clarté sans verser dans le lapidaire. Ce dernier exemple laisse entrevoir une présence phatique, qui répond à la formulation très abstraite en français. Cette connivence serait encore plus patente avec "So, more commuting", voire "More commuting!». Ces énoncés conviendraient peut-être pour un titre de conférence, même savante, sur les infrastructures territoriales, mais ils sont trop expressifs, par leur brièveté même, pour un article de recherche. Il faut résister à la tentation de pousser trop loin la concision en prenant le lecteur en otage, c'est-à-dire de sous-traduire. En l'occurrence, il serait peut-être plus juste de traduire l'exemple 4 par "One result of this trend is increased commuter traffic ».

Ajoutons enfin que l'unité de longueur employée pour un texte en anglais est généralement le nombre de mots, et non le nombre de signes, comme c'est le cas pour d'autres langues. Néanmoins, la concision passe aussi par le lexique. "Prefer the shorter word to the longer», préconise George Orwell (1946). Il est de bonne pratique en anglais de chercher le mot court. On peut ainsi remplacer to centrifuge par to spin, to masticate par to chew, pulmonary par lung, ou assessment criterion par endpoint, selon l'usage. 


\section{Perspectives}

La concision, quand elle n'est ni approximation, ni schématisation, ni raccourci, ni mantra, ni bon mot, quand elle dit le référent, tout le référent et rien que le référent, apporte une valeur ajoutée à la traduction scientifique, valeur appréciée par les auteurs d'articles de recherche, que ce soit en sciences physiques (les STEM des Anglo-Saxons) ou en sciences humaines et sociales. Il en va de même de la révision des textes de plus en plus fréquemment rédigés directement en anglais par des francophones, ou des traductions à caractère interlinéaire, comme celles réalisées par les systèmes de traduction automatique, dont les capacités terminologiques et phraséologiques deviennent pourtant toujours plus impressionnantes. Mais c'est là une autre histoire. II semble peu probable que la machine puisse un jour assumer la totalité des missions d'un professionnel réellement expérimenté. Elle restera un outil, notamment terminologique.

Il appartient au traducteur scientifique de faire valoir ses multiples compétences auprès de sa clientèle. En tant que professionnel de la langue et du langage au service de la communication, aucun champ ne lui en est ni étranger ni interdit. II sait adapter ses prestations à une demande en évolution ou, à l'occasion, en proposer de nouvelles, qui seront autant de sources de valeur ajoutée. Améliorer la qualité d'un texte en le traduisant est normal sinon obligé dans une démarche «cibliste». Or apporter au texte un supplément de concision mesurée, c'est presque toujours l'améliorer lorsqu'on traduit du français vers l'anglais. Reste enfin au professionnel à faire apprécier cette vigilance à sa juste valeur.

\section{richard.ryan@wanadoo.fr}

Richard Ryan est traducteur scientifique spécialisé dans plusieurs domaines de la recherche médicale. Maître de conférences honoraire d'anglais et membre associé du laboratoire de recherche sur le langage à I'université Clermont-Auvergne, il est l'auteur de nombreux articles sur la traduction professionnelle. II enseigne la traduction spécialisée vers I'anglais à I'ISIT, Paris. 


\section{SOURCES CITÉES}

ALLOU Charles-Nicolas, Essai sur l'universalité de la langue française, Paris, Didot, 1828.

Canadian Health Services Research Foundation, Communication notes: putting your readers first, Ottawa, Canadian Health Services Research Foundation, https:// www.cfhi-fcass.ca/Migrated/PDF/CommunicationNotes/cn-selfedit_e.pdf, consulté le 2/06/2020.

FOWLER Henry et FOWLER Francis, The King's English, Oxford, Clarendon Press, 1908.

JAKOBSON Roman, Essais de linguistique générale, Tome I, Les fondation du langage, Paris, Éditions de Minuit, 1963.

ORWELL George, Politics and the English Language, Londres, Horizon, 1946.

RIVAROL de Antoine, De l'universalité de la langue française, Paris, Bailly et Desenne, 1784.

STRUNK William, The Elements of Style, New York, Harcourt, Brace and Howe, 1920.

VANNIER Antonin, La Clarté française, Paris, Nathan, 1923.

VINAY Jean-Paul et DARBELNET Jean, Stylistique comparée du français et de l'anglais, Paris, Didier, 1958. 